\title{
Health Orientation, Knowledge, and Attitudes toward Genetic Testing and Personalized Genomic Services: Preliminary Data from an Italian Sample
}

\author{
Serena Oliveri,, ${ }^{1,2}$ Marianna Masiero, ${ }^{1,2}$ Paola Arnaboldi, ${ }^{2}$ Ilaria Cutica, \\ Chiara Fioretti, ${ }^{2}$ and Gabriella Pravettoni ${ }^{1,2}$ \\ ${ }^{1}$ Interdisciplinary Research Center on Decision Making Processes (IRIDe), Department of Oncology and Hemato-Oncology (DIPO), \\ University of Milan, Via Festa del Perdono 7, 20122 Milan, Italy \\ ${ }^{2}$ Applied Research Division for Cognitive and Psychological Science, Istituto Europeo di Oncologia (IEO), \\ Via Ripamonti 435, 20141 Milan, Italy
}

Correspondence should be addressed to Serena Oliveri; serena.oliveri@unimi.it

Received 1 September 2016; Revised 14 November 2016; Accepted 12 December 2016

Academic Editor: Joseph Telfair

Copyright (C) 2016 Serena Oliveri et al. This is an open access article distributed under the Creative Commons Attribution License, which permits unrestricted use, distribution, and reproduction in any medium, provided the original work is properly cited.

\begin{abstract}
Objective. The study aims at assessing personality tendencies and orientations that could be closely correlated with knowledge, awareness, and interest toward undergoing genetic testing. Methods. A sample of 145 subjects in Italy completed an online survey, investigating demographic data, health orientation, level of perceived knowledge about genetic risk, genetic screening, and personal attitudes toward direct to consumer genetic testing (DTCGT). Results. Results showed that respondents considered genetic assessment to be helpful for disease prevention, but they were concerned that results could affect their life planning with little clinical utility. Furthermore, a very high percentage of respondents (67\%) had never heard about genetic testing directly available to the public. Data showed that personality tendencies, such as personal health consciousness, health internal control, health esteem, and confidence, motivation to avoid unhealthiness and motivation for healthiness affected the uptake of genetic information and the interest in undergoing genetic testing. Conclusions. Public knowledge and attitudes toward genetic risk and genetic testing among European countries, along with individual personality and psychological tendencies that could affect these attitudes, remain unexplored. The present study constitutes one of the first attempts to investigate how such personality tendencies could motivation to undergo genetic testing and engagement in lifestyle changes.
\end{abstract}

\section{Introduction}

Preemptive medicine [1] and the recent progress in genomics bear the promise that the identification of individual traits, risk factors, and the prediction of latent diseases may allow the development of personalized interventions (e.g., the most efficient drug, with the right dose with the right time-using) and thus decrease the probability of disease development [2]. There are currently genetic tests for over 1,500 diseases, a number of which are directly available to the public for personal use; in the early 1980s several private companies started to offer genetic testing directly to the citizens (DTCGT). This situation raises concerns on risk communication, health responsibility between clinicians and patients, and management of the gap between genetic susceptibility and lack of therapeutic options [3-5]. Nevertheless, we cannot neglect the fact that genetic information is now an integral part of risk assessment and that it constitutes a resource for individuals in some cases [6], who may decide to cope with manageable factors (e.g., smoking, diet, exercise, and alcohol intake), opt for specific prophylactic treatments or clinical screenings, and rearrange future plans (e.g., decisions for the offspring) (WHO).

Healthcare professionals and policy makers in Europe are struggling to find effective strategies to communicate genetic risk information and, at the same time, to enhance individual empowerment and shared decision making in this field [7].

A considerable amount of studies have investigated the level of knowledge, attitudes, and ability to manage genetic 
information among general practitioners [8-12] and the interest of people in genetic testing in many European countries [13-21]. They attempted to provide a global view of the general public's predisposition to consider and undergo genetic testing, but to date no studies have tried to correlate these trends with specific psychological tendencies associated with health [22-24]. Previous literature in the US [25], instead, put emotional reactions to genetic risk information in the limelight, but as it focuses almost uniquely on anxiety and depression, it failed to capture the most complex psychological, cognitive, and emotional processes which can act as motivators for health knowledge acquisition and can become the precursors of patients' health engagement and behavioral change [26]. Hence, the aim of this study was to assess the personality tendencies that could be closely correlated with knowledge, awareness, and interest in undergoing genetic testing in a sample of the Italian population. We choose a sample of Italian citizens, prevalently young adults and adults [27], with higher education, according to the cultural standards and the education system in Italy, since previous studies showed that this cohort of people have more open attitudes towards new health technologies, are more interested in scientific progress, and are more confident with the use of Internet services and platforms (which nowadays are the most powerful source for information) $[13,15,28-30]$.

\section{Materials and Methods}

2.1. Participants and Procedure. A total of 250 subjects with at least a bachelor degree were invited by e-mail to complete the survey. They were recruited through authors' social networks and students' institutional mailing-list via the University of Milan (Italy) from September 2015 to January 2016. A sample of 145 subjects agreed to complete our survey; $22.1 \%$ were male (32), whereas $77.9 \%$ were female (113). The mean age was $31.41(\mathrm{SD}=7.586)($ minimum $=21$ and maximum $=54)$ and among them $49 \%$ had a bachelor's degree, whereas $51 \%$ ranged from master degree to Ph.D. and/or specialization (4 years of training after master degree); $16 \%$ of the participants had children.

Each participant could contact the researcher in order to receive clarifications about the scope and the methodology of the study. After this primary contact researcher sent a link with the questionnaire. Data were collected using Survey Monkey, an open source online survey application which enables users to develop and publish online surveys and register responses (www.surveymokey.com). Overall, the time required for filling in the questionnaire was 20 minutes. Participation to the study was voluntary and anonymous. The research protocol was approved by the Institutional Review Board of the University of Milan.

2.2. Measures. A structured and self-administered ad hoc questionnaire was completed by the enrolled participants. It was divided into different sections.

(i) Demographic Variables. The questionnaire included a set of specific questions designed to depict sociodemographic characteristics such as gender, age, marital status, number of offspring, educational level, and current employment. Furthermore, two parameters of physical status were included (weight and height for BMI classification).

(ii) Health Orientation Scale (HOS) [31]. It is a self-administered questionnaire that assesses several health-related personality features. It is composed of fifty items divided into ten subscales: personal health consciousness (the tendency to be highly aware of one's physical health); health image concern (the tendency to be highly aware of the external, observable impression that one's physical health makes on others); health anxiety (the tendency to be anxious about one's physical health); health esteem and confidence (the tendency to feel confident about one's physical health); motivation to avoid unhealthiness (the desire to avoid being in a state of unhealthiness); motivation for healthiness (the desire to keep oneself in excellent physical health); health internal control (the tendency to believe that one's physical health is dependent upon one's behaviors); health external control (the tendency to believe that one's health status is determined by factors that do not depend upon ones' actions); health expectations (the tendency to expect that one's health will be excellent and positive in the future); health status (the tendency to regard oneself as currently being in good physical shape). These personality features are intended as inner states which potentially affect thinking, feelings, and behaviors.

Respondents were asked to indicate the extent to which they identify with each statement on a 5-point Likert scale $(1=$ not at all characteristic of me, $2=$ slightly characteristic of me, 3 = somewhat characteristic of me, $4=$ moderately characteristic of me, and $5=$ very characteristic of me). Total scoring for each subscale was $0-20$. The internal consistency was evaluated using Cronbach alpha coefficient: subscales reported a value included within 0.69-0.92. However, the Spearman-Brown coefficient was used to control the reliability: subscales reported a value included within 0.82-0.96 [31].

(iii) Genetic Knowledge and Attitudes toward DTCGT. In order to evaluate the level of perceived knowledge about genetic risk, genetic testing and DTCGT services, and the implications for future health behaviors and decisions, respondents were asked a set of questions selected and adapted from previous studies $[15,32]$. In particular, we considered an European survey conducted on 725 young adults and adults on awareness and knowledge about DTCGT [15]. Thus comparisons could be made between European populations available. Questions assessed the following pivotal points:

(a) The level of perceived knowledge about genetic risk ("What is your level of knowledge about genetic risk?" evaluated using a Likert scale: poor, fair, good, and excellent), genetic testing ("What is your level of knowledge about genetic testing?" evaluated using a Likert scale: poor, fair, good, and excellent), and DTCGT ("Have you ever heard about genetic tests that are provided directly to consumers via the Internet (DTC)?" evaluated with a dichotomous response yes or no)

(b) The sources of information ("Where did you gather information?" open question) 
(c) The motivation for accepting and/or for refusing the DTCGT ("For which reason would you consider to undergo a genetic analysis direct to consumer via the Internet?" evaluated through listed options), with the possible impact on current and/or future health behaviors and decisions (items options "I think it might influence my difficulty in making decisions concerning my health," "I think it can influence my future decisions" (e.g., decisions related to marriage, pregnancy, offspring etc.)

(d) The intention to undergo the DTCGT (rated on a scale from 0 , not at all motivated, to 10 , highly motivated).

2.3. Statistical Analysis. A descriptive analysis was conducted to report demographic, social, and professional characteristics of the subjects enrolled. A multivariate analysis of variance (MANOVA) was calculated using the overall level of perceived knowledge about genetic risk and genetic testing (poor, fair, good, and excellent) or genomic medicine and DTCGT (yes, no) as fixed factor and health-related personality tendency (HOS subscales) as dependent variable (2tailed, $p<.05)$. The Pearson product-moment coefficient was used to measure the correlation between intention of the subjects to undergo genetic testing and their health-related personality tendency (2-tailed, $p<.05)$, considering all ten subscales of HOS. Analyses were performed including all the subjects with the SPSS package (version 20.0, IBM, USA, 2014).

\section{Results}

3.1. Demographic Data. Considering the parameters on physical status, our sample had a mean height of $1.69 \mathrm{~m}(\mathrm{SD}=$ $.0815)$ and a mean weight of $63.92 \mathrm{~kg}(\mathrm{SD}=16.382)$. According to the international classification the sample had a normal BMI $(\mathrm{M}=22.11 \mathrm{SD}=4.246)$.

No differences in attitudes and perceived knowledge toward genetic risk, screening, and direct to consumer genetic testing were found with regard to sex, age (aged 21 to 30 , aged 31 to 40 , and aged 41 to 54 ), physical status (categories according to international BMI classification), and educational level (bachelor degree, master degree, post degree specialization).

3.2. Genetic Knowledge and Attitudes toward Genetic Risk, Genetic Testing, and DTCGT. Participants declared that they had an adequate level of information about genetic risk; indeed, only $9 \%$ of the subjects stated having poor information, while $40 \%$ and $38.6 \%$ declared having a fair or good level of knowledge (see Figure 1). Regarding the perceived knowledge of genetic testing, a more consistent distribution was observed among all answer options (poor 24.1\%; fair 28.3\%; good 36.7\%; excellent 7.6\%) (see Figure 2). Two dichotomous questions evaluating the familiarity with personalized genomic medicine and the existence of DTCGT were used. Subjects were equally distributed among the following: "I know genomic medicine" (51.7\%) and "I do

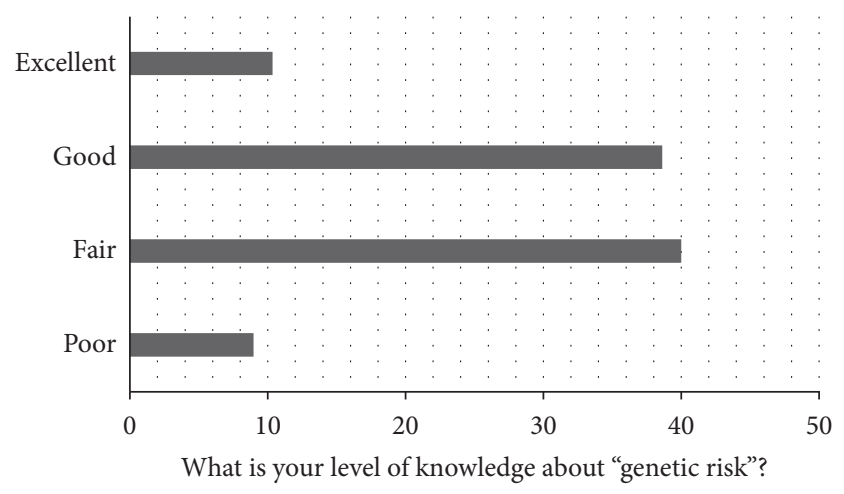

Figure 1: Genetic risk knowledge.

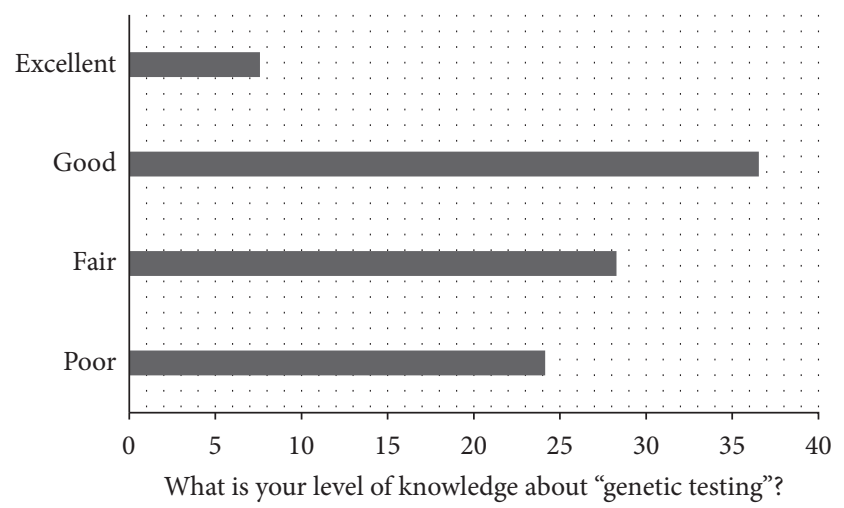

Figure 2: Genetic testing knowledge.

not know genomic medicine" (46.7\%). Different results were observed for DTCGT: subjects who never heard about direct to consumer genetic testing (66.2\%) were twice as much as people who were aware of DTCGT existence (33\%).

3.3. Motivations to Undergo and to Reject DTCGT. Among participants who declared that they knew about DTCGT, the foremost reasons to get DTCGT were mainly connected with health-related motivations and expectations. Thus, "knowing my current health status" (14.4\%), "adopting health behaviors" (15.2\%), and "increasing early detection of the disease" (21.4\%) were the most frequent answers (see Figure 3). Interestingly, two motivations guided the idea of rejecting the DTCGT: "the results might cause worry, interfering with future behaviors" (17.2\%) and "the results are unreliable" (24.2\%) (see Figure 4).

3.4. Sources of Information for Genetic Risk and DTCGT. The chief sources of information for both genetic risk (45.5\%) and DTCGT (41.4\%) were the work-education system, while other different sources of information mentioned were approximately equally distributed among participants' judgments. Data indicated that secondary sources of information for genetic risk were the mass-media (10.3\%) and Internet (9.7\%), whereas those for DTCGT were scientific journals (11\%) and Internet (6.2\%) (see Figures 5 and 6). 


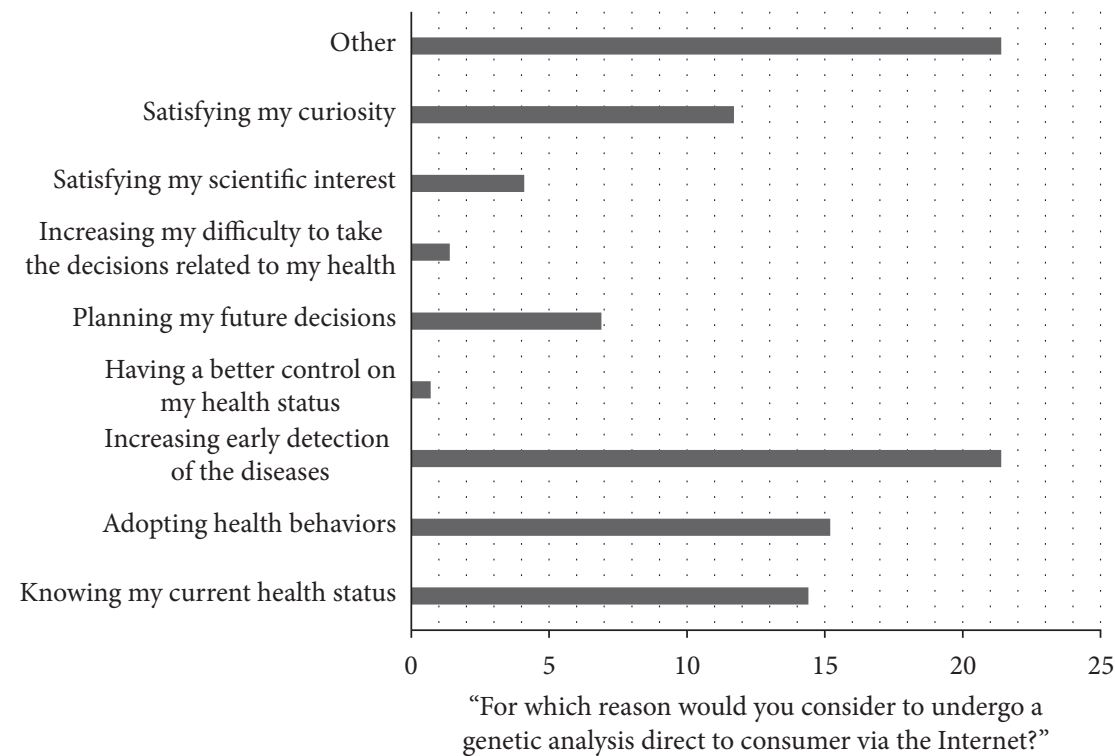

FIGURE 3: Reasons to use DTCGT.

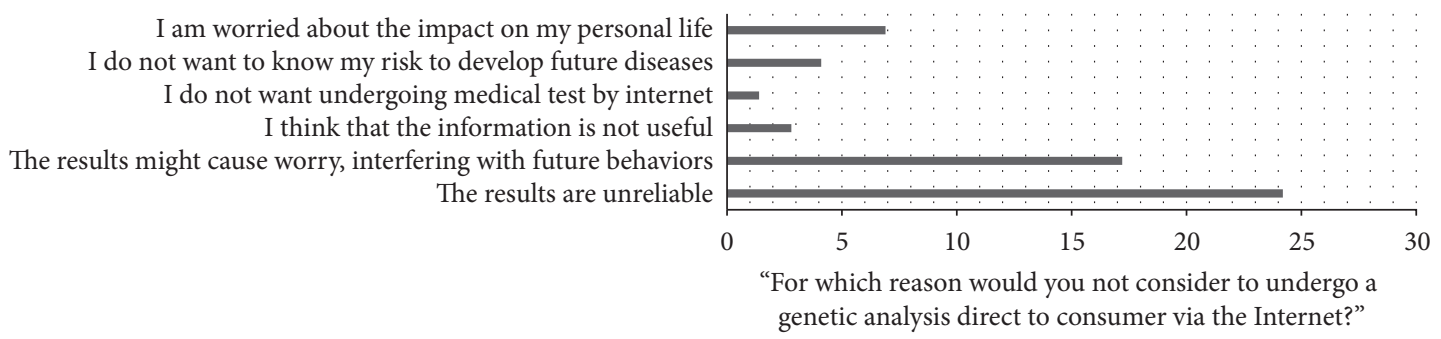

FIGURE 4: Reasons to reject DTCGT.

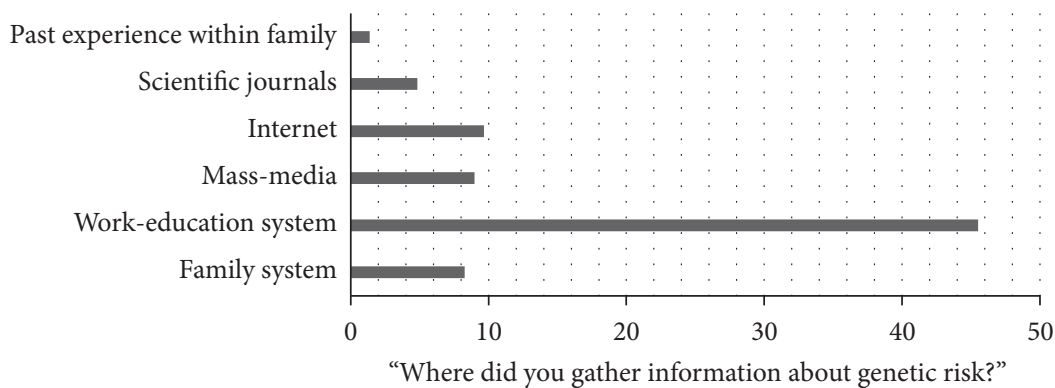

FIGURE 5: Sources on genetic risk.

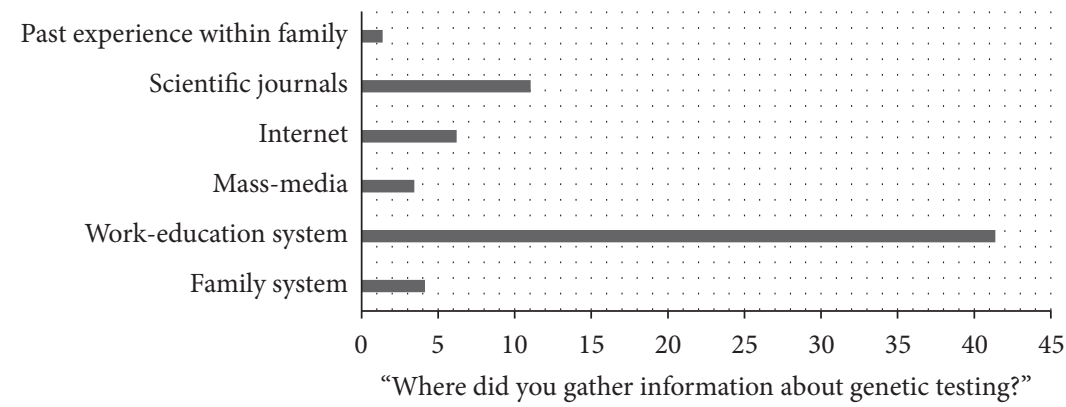

FIgURE 6: Sources on DTCGT. 
TABLE 1: MANOVA between the level of perceived knowledge of genetic risk and the HOS subscales scores.

\begin{tabular}{|c|c|c|c|c|c|c|c|c|}
\hline & Health orientation scale (HOS) & df & Mean square & $F$ & Sig. & $\begin{array}{c}\text { Partial eta } \\
\text { squared }\end{array}$ & $\begin{array}{l}\text { Noncent. } \\
\text { parameter }\end{array}$ & $\begin{array}{c}\text { Observed } \\
\text { power }\end{array}$ \\
\hline \multirow{2}{*}{ Genetic risk knowledge } & Personal health consciousness (PHC) & 3 & 41.854 & 4.163 & .007 & .085 & 11.997 & .828 \\
\hline & Health internal control (HIC) & 3 & 48.188 & 3.817 & .012 & .082 & 11.566 & .812 \\
\hline
\end{tabular}

TABle 2: Post hoc Bonferroni test.

\begin{tabular}{|c|c|c|c|c|c|c|c|}
\hline & Geneti & nowledge & Mean difference $(I-J)$ & Std. error & Sig. & Lower bound & Upper bound \\
\hline \multirow{2}{*}{ Personal health consciousness ( $\mathrm{PHC}$ ) } & \multirow{2}{*}{ Poor } & Fair & -3.06 & .996 & .015 & -5.73 & -.39 \\
\hline & & Good & -3.95 & 1.226 & .010 & -7.23 & -.66 \\
\hline \multirow{2}{*}{ Health internal control (HIC) } & \multirow{2}{*}{ Poor } & Fair & -3.00 & 1.103 & .044 & -5.96 & -.05 \\
\hline & & Good & -4.19 & 1.340 & .013 & -7.78 & -.60 \\
\hline
\end{tabular}

TABLE 3: MANOVA between the level of perceived knowledge on genetic testing and HOS subscales scores.

\begin{tabular}{lcccccccc}
\hline & Health orientation scale (HOS) & df. & $\begin{array}{c}\text { Mean } \\
\text { square }\end{array}$ & $F$ & Sig. & $\begin{array}{c}\text { Partial eta } \\
\text { squared }\end{array}$ & $\begin{array}{c}\text { Noncent. } \\
\text { parameter }\end{array}$ & $\begin{array}{c}\text { Observed } \\
\text { power }\end{array}$ \\
\hline & Personal health consciousness (PHC) & 3 & 39.051 & 3.931 & .010 & .079 & 11.223 & .800 \\
DTCGT knowledge & Motivation to avoid unhealthiness (MAU) & 3 & 41.896 & 3.760 & .012 & .066 & 9.196 & .707 \\
& Motivation for healthiness (MH) & 3 & 62.921 & 4.303 & .006 & .083 & 11.879 & .824 \\
& Health internal control (HIC) & 3 & 57.439 & 4.551 & .005 & .100 & 14.533 & .898 \\
\hline
\end{tabular}

TABLE 4: Post hoc Bonferroni test.

\begin{tabular}{|c|c|c|c|c|c|c|c|}
\hline \multirow{2}{*}{ Personal health consciousness (PHC) } & \multicolumn{2}{|c|}{ DTCGT knowledge } & \multirow{2}{*}{$\begin{array}{c}\text { Mean difference }(I-J) \\
-2.21\end{array}$} & \multirow{2}{*}{$\begin{array}{c}\begin{array}{c}\text { Std. } \\
\text { error }\end{array} \\
.767\end{array}$} & \multirow{2}{*}{$\begin{array}{l}\text { Sig. } \\
.028\end{array}$} & \multicolumn{2}{|c|}{$\begin{array}{c}95 \% \text { confidence interval } \\
\text { Lower bound Upper bound }\end{array}$} \\
\hline & Poor & Fair & & & & -4.26 & -.15 \\
\hline Motivation to avoid unhealthiness (MAU) & Poor & Fair & -2.32 & .878 & .054 & -4.68 & .03 \\
\hline Motivation for healthiness (MH) & Poor & Fair & -2.87 & .946 & .017 & -5.41 & -.34 \\
\hline \multirow{2}{*}{ Health internal control (HIC) } & \multirow{2}{*}{ Poor } & Fair & -2.81 & .817 & .005 & -5.00 & -.62 \\
\hline & & Good & -3.21 & 1.190 & .047 & -6.40 & -.02 \\
\hline
\end{tabular}

3.5. Health Attitudes That Affect the Knowledge about Genetic Risk and DTCGT. A multivariate analysis of variance (MANOVA) between subjects was used to evaluate whether specific personality attitudes might affect the tendency to get information about genetic risk and genetic testing.

The level of perceived knowledge of genetic risk (poor, fair, good, and excellent) was set as a fixed factor, distinguishing groups of subjects based on the level of perceived genetic knowledge, and the HOS subscales scores as dependent variables. Statistically significant differences among groups were found for two of the HOS subscales: personal health consciousness (PHC) and health internal control (HIC). Data showed that subjects who were better informed on genetic risk had a higher level of PHC (Wilks's $\Lambda=.015 ; F(3)=$ $4.163 p=.007$ ) and HIC (Wilks's $\Lambda=.015 ; F(3)=3.817 p=$ .012) (see Tables 1 and 2).

A separate MANOVA was applied to examine the association between the level of perceived knowledge on genetic testing (poor, fair, good, and excellent), used as a fixed factor, and HOS subscales scores as dependent variables.
Statistically significant differences among groups were found for four HOS subscales: personal health consciousness (PHC) (Wilks's $\Lambda=.013 ; F(3)=3,931 p=.010)$; health internal control (HIC) (Wilks's $\Lambda=.013 ; F(3)=4.551 p=.005$ ); motivation to avoid unhealthiness (MAU) (Wilks's $\Lambda=.013$; $F(3)=3.760 p=.012)$; motivation for healthiness $(\mathrm{MH})$ (Wilks's $\Lambda=.013 ; F(3)=4.303 p=.006$ ) (see Tables 3 and 4). It was observed that higher levels in these subscales were associated with better reported knowledge on genetic testing.

3.6. Health Attitudes That Affect the Intention to Undergo to the DTCGT. The Pearson product-moment correlation coefficient was used to measure the association between subjects' health-related personality features and the intention to undergo DTCGT (0, not at all motivated, to 10 , highly motivated). health esteem and confidence subscales were negatively correlated to the intention to undergo the DTCGT $\left(X^{2}=-.208 p=.014\right)$. Subjects who score high in this subscale tend to report a high self-confidence toward their health status. 


\section{Discussion}

Genomic medicine is a major step forward for the medical and scientific community. Broadly speaking, public attitudes towards genetic testing in Europe are positive [17-19, 32-34]. They tend to differ by country and in relation to individual features such as age, gender, and educational level $[33,35]$. For instance, in the UK [13], Greece [15], and Finland $[17,33]$, younger people and males reported a higher level of interest in genetic testing and DTCGT, along with a more open attitude towards new health technologies [27]. In the Netherlands [18] a significant difference between lower and higher-educated people emerged, with lower-educated respondents showing more interest in genetic testing than higher-educated respondents. In our population, which represents a specific age and educational range of the Italian population (see Participants and Procedure), we verified if there should be significant differences based on the single instructional titles and based on three different decades. Overall, we found no statistical differences of perceived knowledge toward genetic risk, testing, DTCGT, and attitudes towards undergoing genetic testing based on sex, age, physical status, and educational level.

Our results show that, overall, participants claimed to have a good knowledge about genetic risk and genetic testing, although more than half of them never heard about DTCGT (66.2\%). This low rate of knowledge about DTCGT was also observed in studies conducted in the UK [13], where the level of awareness among the general public was about $13 \%$, and Greece [15], with only one-third of the population aware of DTCGT.

Our respondents considered genetic assessment to be helpful for disease prevention and for gathering information on their health status and disease detection, but they were concerned that results could affect their life planning with little clinical utility. Our results are in accordance with previous studies conducted on similar cohorts of subjects in Europe, which showed that better knowledge about genetics did not simply lead to unambiguous acceptance of genetic testing and that a considerable proportion of people in Europe have some worries and doubts [13, 17, 32, 33, 36, 37]. The most popular reasons to approve genetic testing in Europe were that it is useful to adopt a healthier lifestyle and live longer $[13,19,36]$, to learn more about one's health status, and to find out one's disease risk $[13,15,30]$. Instead the most mentioned reasons for refusal to take up genetic testing, DTCGT in particular, were that genetic testing should be performed mainly in the hospital or with a physician's mediation and/or it should be directed towards curable or preventable diseases $[15,18,37]$ since it might cause people to become excessively worried or would impact future plans about offspring $[30,33,36]$.

In our sample as well, most of respondents claimed to have specific concerns about DTCGT, such as "the results are unreliable" and "the results might cause worry interfering with future behaviors." We argue that these results could be read in light of the fact that in Italy genetic tests are primarily offered within health care systems, and most respondents believe that it is how they should continue to be offered, that is, in regular healthcare contexts (such as the hospital), which are able to guarantee the needed quality check.

Interestingly, one reason frequently reported in the literature among the motivations to undergo DTCGT is interest in the health status of one's children [38, 39], such as learning about their children's risk [40]. Conversely, we found that none of our participants addressed this issue. This might be due to the lack of a specific item addressing this issue into our survey (despite the presence of "other reasons" among the options), or the lack of knowledge about what kind of information genetic testing can provide the health status of one's offspring, or because they just do not want to know the genetic condition of their offspring.

Findings from other studies have reported that if the media pay serious attention to genetics, then people's expectations of the benefits of genetic testing increase, as does its use [19]. In our population, about half of the respondents recognized the work and educational domain as the best information source in the area of genetic risk ( $41 \%$ of participants) and genetic screening and genetic services (45.5\%). Thus the school or academic fields are the primary source for information about genetics, while Internet and overall mass media are only a secondary source for genetic information and genetic services disclosure. This could explain why DTCGT is virtually unknown among our targeted population, compared to many other countries: DTCGT is largely advertised on the private companies' web sites $[41,42]$ that are not the favored genetic information sources for our population.

We investigated which personality attitudes toward health could influence the uptake of genetic information, genetic knowledge, and awareness and the interest in undergoing genetic testing. Interestingly our results showed that people who are more informed about genetic risk and genetic testing have a high personal health consciousness (PHC) and a health internal control (HIC). Health consciousness is a multifaceted concept [43-45], and it could be explained as the tendency to think about one's physical health and fitness [31]. PHC is, first of all, a psychological or inner status [45] which includes health alertness, health involvement, and selfmonitoring [46, 47], different from health anxiety or fear of being sick or of dying. In our sample people who were more predisposed to devote attention to their health status (high PHC) were more interested and predisposed to gather info about genetic risk, genetic testing, and even DTCGT; these later prevalently spread by mass media channels (Internet). Participants with more knowledge about DTCGT had, moreover, high motivation to avoid unhealthiness (MAU) and motivation for healthiness (MH). This means that they are more predisposed to engage with active solutions and healthrelated behaviors such as information seeking; they are active in avoiding behaviors and activities which undermine their physical health and preserve their wellness [48].

Another important personal component, which proved to be related to genetic knowledge and interest, was the Health Locus of Control (HLC). HLC is the extent to which individuals believe that their health is determined by their own actions or by environmental circumstances and powerful external agents and thus whether it is manageable or not $[49,50]$. An internal locus of control suggests that one's health 
outcome is under the direct control of one's own actions. In general, people who believe that they could influence their health status with health-related behaviors are more likely to avoid health risks and adopt preventive solutions. As outlined before, our results show that participants with high HIC (health internal control) gather more information about what actual scientific progress has to offer in the domain of prevention and personalized treatments, such as genetic testing including DTCGT.

We asked participants who claimed to have a good knowledge of DTCGT whether they would be interested in undergoing DTCGT. Participants who were more interested in undergoing DTCGT had low levels of health esteem and confidence, a measure of how much someone is feeling good about himself/herself, believing in himself/herself and his/her wellness. This could mean that the more a person is critical about his/her physical wellness, the more he/she is interested in undergoing genetic testing even if there is no medical prescription, believing that such kind of information could reassure them about their health or give therapeutic solutions. This kind of tendencies could lead to inappropriate and useless health-related decisions.

\section{Conclusions}

We can summarize that the population of Italian respondents has a rather poor knowledge of genetic medicine and of DTCGT. Also, they seem to mostly rely on work and educational domain to gain information about such topics. As a consequence, in the need of increasing the population literacy and the possibility to make informed decisions on genetic matters [6], it could be useful to convey such information in a way that would be perceived as reliable (e.g., through institutional programs).

However, in order to embed genetic risk information and genetic testing in health programs, we need to understand people's psychological traits, attitudes, beliefs, and decision making processes [51-53] that could potentially influence the motivation to search for genomic risk information, decision to undergo genetic testing, and engaging in lifestyle changes.

The present study is a first attempt to assess personality tendencies and orientations that could be correlated with knowledge, awareness, and interest toward undergoing genetic testing. We found that some measurable characteristics, such as high personal consciousness, and health internal control were correlated to interest and knowledge on genetic matters and to the preferred way to search for information. However, this promising research topic needs to be investigated more deeply.

Finally, we recognize two possible limitations of our study: the selection of participants did not include elderly people (in order to guarantee the participation of computerliterate individuals) and participants were not "real" consumers but we asked for their hypothetical interest in DTCGT and genetic testing in general.

\section{Ethical Approval}

This manuscript complies with the Ethical Rules applicable for this journal. The research protocol was approved by the
Institutional Review Board of the University of Milan, the principal coordination center of the research. The study was conducted according to Helsinki declaration.

\section{Consent}

In line with the University of Milan ethical procedures, participants completed a brief informed consent form for this observational study.

\section{Competing Interests}

The authors declare no competing interests.

\section{Authors' Contributions}

Serena Oliveri and Marianna Masiero contributed equally to this work.

\section{Acknowledgments}

This work is supported by a grant for the project "Mind the Risk" from The Swedish Foundation for Humanities and Social Sciences, which had no influence on the content of this paper (Grant no. M13-0260:1).

\section{References}

[1] S. J. Bielinski, J. E. Olson, J. Pathak et al., "Preemptive genotyping for personalized medicine: design of the right drug, right dose, right time-using genomic data to individualize treatment protocol," Mayo Clinic Proceedings, vol. 89, no. 1, pp. 25-33, 2014.

[2] N. S. Abul-Husn, A. Owusu Obeng, S. C. Sanderson, O. Gottesman, and S. A. Scott, "Implementation and utilization of genetic testing in personalized medicine," Pharmacogenomics and Personalized Medicine, vol. 7, no. 1, pp. 227-240, 2014.

[3] C. A. Shelton and D. C. Whitcomb, "Evolving roles for physicians and genetic counselors in managing complex genetic disorders," Clinical and Translational Gastroenterology, vol. 6, no. 11, p. e124, 2015.

[4] K. F. L. Douma, E. M. A. Smets, and D. C. Allain, "Non-genetic health professionals' attitude towards, knowledge of and skills in discussing and ordering genetic testing for hereditary cancer," Familial Cancer, vol. 15, no. 2, pp. 341-350, 2016.

[5] M. Weaver, "The double helix: applying an ethic of care to the duty to warn genetic relatives of genetic information," Bioethics, vol. 30, no. 3, pp. 181-187, 2016.

[6] S. Oliveri, G. Pravettoni, C. Fioretti, and M. G. Hansson, "Let the individuals directly concerned decide: a solution to tragic choices in genetic risk information," Public Health Genomics, vol. 19, no. 5, pp. 307-313, 2016.

[7] F. Becker, C. G. Van El, D. Ibarreta et al., "Genetic testing and common disorders in a public health framework: how to assess relevance and possibilities," European Journal of Human Genetics, vol. 19, no. 1, pp. S6-S44, 2011. 
[8] M. J. H. Baars, L. Henneman, and L. P. Ten Kate, "Deficiency of knowledge of genetics and genetic tests among general practitioners, gynecologists, and pediatricians: a global problem," Genetics in Medicine, vol. 7, no. 9, pp. 605-610, 2005.

[9] K. Challen, H. Harris, U. Kristoffersson et al., "General practitioner management of genetic aspects of a cardiac disease: a scenario-based study to anticipate providers' practices," Journal of Community Genetics, vol. 1, no. 2, pp. 83-90, 2010.

[10] Z. Klemenc-Ketiš and B. Peterlin, "Family physicians' management of genetic aspects of a cardiac disease: a scenario-based study from Slovenia," Balkan Journal of Medical Genetics, vol. 17, no. 1, pp. 15-22, 2014.

[11] E. J. Houwink, S. J. Van Luijk, L. Henneman, C. Van Der Vleuten, G. Jan Dinant, and M. C. Cornel, "Genetic educational needs and the role of genetics in primary care: a focus group study with multiple perspectives," BMC Family Practice, vol. 12, 2011.

[12] H. C. Howard and P. Borry, "Survey of European clinical geneticists on awareness, experiences and attitudes towards direct-to-consumer genetic testing," Genome Medicine, vol. 5, no. 5, article no. 45, 2013.

[13] L. F. Cherkas, J. M. Harris, E. Levinson, T. D. Spector, and B. Prainsack, "A survey of UK public interest in internet-based personal genome testing," PLoS ONE, vol. 5, no. 10, Article ID e13473, 2010.

[14] E. Vayena, E. Gourna, J. Streuli, E. Hafen, and B. Prainsack, "Experiences of early users of direct-to-consumer genomics in Switzerland: an exploratory study," Public Health Genomics, vol. 15 , no. 6, pp. 352-362, 2012.

[15] V. Mavroidopoulou, E. Xera, and V. Mollaki, "Awareness, attitudes and perspectives of direct-to-consumer genetic testing in Greece: a survey of potential consumers," Journal of Human Genetics, vol. 60, no. 9, pp. 515-523, 2015.

[16] P. Jallinoja and A. R. Aro, "Does knowledge make a difference? The association between knowledge about genes and attitudes toward gene tests," Journal of Health Communication, vol. 5, no. 1, pp. 29-39, 2000.

[17] M. Hietala, A. Hakonen, A. R. Aro, P. Niemela, L. Peltonen, and P. Aula, "Attitudes toward genetic testing among the general population and relatives of patients with a severe genetic disease: a survey from Finland," American Journal of Human Genetics, vol. 56, no. 6, pp. 1493-1500, 1995.

[18] E. Vermeulen, L. Henneman, C. G. Van El, and M. C. Cornel, "Public attitudes towards preventive genomics and personal interest in genetic testing to prevent disease: a survey study," The European Journal of Public Health, vol. 24, no. 5, pp. 768-775, 2013.

[19] L. Henneman, E. Vermeulen, C. G. van El et al., "Public attitudes towards genetic testing revisited: comparing opinions between 2002 and 2010," European Journal of Human Genetics, vol. 21, no. 8, pp. 793-799, 2002.

[20] N. Panic, E. Leoncini, P. Di Giannantonio et al., "Survey on knowledge, attitudes, and training needs of Italian residents on genetic tests for hereditary breast and colorectal cancer," BioMed Research International, vol. 2014, Article ID 418416, 7 pages, 2014.

[21] H. Calsbeek, M. Morren, J. Bensing, and M. Rijken, "Knowledge and attitudes towards genetic testing: a two year follow-up study in patients with asthma, diabetes mellitus and cardiovascular disease," Journal of Genetic Counseling, vol. 16, no. 4, pp. 493$504,2007$.
[22] S. Oliveri, C. Renzi, and G. Pravettoni, “Toward an in-depth profiling of DTC users," Clinical Genetics, vol. 88, no. 5, pp. 505506, 2015.

[23] S. Oliveri, C. Renzi, M. Masiero, and G. Pravettoni, "Living at risk: factors that affect the experience of direct-to-consumer genetic testing," Mayo Clinic Proceedings, vol. 90, no. 10, article no. 1101, pp. 1323-1326, 2015.

[24] S. Oliveri and G. Pravettoni, "The disclosure of direct-toconsumer genetic testing: sounding out the psychological perspective of consumers," Biology and Medicine, vol. 8, article 316, 2016.

[25] J. T. Heshka, C. Palleschi, H. Howley, B. Wilson, and P. S. Wells, "A systematic review of perceived risks, psychological and behavioral impacts of genetic testing," Genetics in Medicine, vol. 10, no. 1, pp. 19-32, 2008.

[26] S. Oliveri, H. C. Howard, C. Renzi, M. G. Hansson, and G. Pravettoni, "Anxiety delivered direct-to-consumer: are we asking the right questions about the impacts of DTC genetic testing?" Journal of Medical Genetics, vol. 53, no. 12, pp. 798799, 2016.

[27] J. J. Arnett, "Emerging adulthood: what is it, and what is it good for?" Child Development Perspectives, vol. 1, no. 2, pp. 68-73, 2007.

[28] G. Gaskell, N. Allum, M. Bauer et al., "Biotechnology and the European public," Nature Biotechnology, vol. 18, no. 9, pp. 935938, 2000.

[29] K. E. Ormond, L. Hudgins, J. M. Ladd, D. M. Magnus, H. T. Greely, and M. K. Cho, "Medical and graduate students' attitudes toward personal genomics," Genetics in Medicine, vol. 13, no. 5, pp. 400-408, 2011.

[30] E. Vayena, C. Ineichen, E. Stoupka, and E. Hafen, "Playing a part in research? University students' attitudes to direct-toconsumer genomics," Public Health Genomics, vol. 17, no. 3, pp. 158-168, 2014.

[31] W. E. Snell, G. Johnson, P. J. Lloyd, and M. W. Hoover, “The health orientation scale: a measure of psychological tendencies associated with health," European Journal of Personality, vol. 5, no. 2, pp. 169-183, 1991.

[32] H. Berth, F. Balck, and A. Dinkel, "Attitudes toward genetic testing in patients at risk for HNPCC/FAP and the German population," Genetic Testing, vol. 6, no. 4, pp. 273-280, 2002.

[33] A. R. Aro, A. Hakonen, M. Hietala et al., "Acceptance of genetic testing in a general population: age, education and gender differences," Patient Education and Counseling, vol. 32, no. 1-2, pp. 41-49, 1997.

[34] C. M. Condit, "Public attitudes and beliefs about genetics," Annual Review of Genomics and Human Genetics, vol. 11, pp. 339-359, 2010.

[35] L. Henneman, D. R. M. Timmermans, and G. Van Der Wal, "Public attitudes toward genetic testing: perceived benefits and objections," Genetic Testing, vol. 10, no. 2, pp. 139-145, 2006.

[36] P. Jallinoja, A. Hakonen, A. R. Aro et al., "Attitudes towards genetic testing: analysis of contradictions," Social Science and Medicine, vol. 46, no. 10, pp. 1367-1374, 1998.

[37] Y. Mai, T. Koromila, A. Sagia et al., "A critical view of the general public's awareness and physicians' opinion of the trends and potential pitfalls of genetic testing in Greece," Personalized Medicine, vol. 8, no. 5, pp. 551-561, 2011.

[38] M. D. Schwartz, J. Benkendorf, C. Lerman, C. Isaacs, A. RyanRobertson, and L. Johnson, "Impact of educational print materials on knowledge, attitudes, and interest in BRCA1/BRCA2: 
testing among Ashkenazi Jewish women," Cancer, vol. 92, no. 4, pp. 932-940, 2001.

[39] H. Chaliki, S. Loader, J. C. Levenkron, W. Logan-Young, W. J. Hall, and P. T. Rowley, "Women's receptivity to testing for a genetic susceptibility to breast cancer," American Journal of Public Health, vol. 85, no. 8, part 1, pp. 1133-1135, 1995.

[40] M. Bruno, S. Tommasi, B. Stea et al., "Awareness of breast cancer genetics and interest in predictive genetic testing: a survey of a southern Italian population," Annals of Oncology, vol. 15, supplement 1, pp. i48-i54, 2004.

[41] C. R. Lachance, L. A. H. Erby, B. M. Ford, V. C. Allen, and K. A. Kaphingst, "Informational content, literacy demands, and usability of websites offering health-related genetic tests directly to consumers," Genetics in Medicine, vol. 12, no. 5, pp. 304-312, 2010.

[42] A. Singleton, L. H. Erby, K. V. Foisie, and K. A. Kaphingst, "Informed choice in direct-to-consumer genetic testing (DTCGT) websites: a content analysis of benefits, risks, and limitations," Journal of Genetic Counseling, vol. 21, no. 3, pp. 433439, 2012.

[43] F. B. Kraft and P. W. Goodell, "Identifying the health conscious consumer," Journal of Health Care Marketing, vol. 13, no. 3, pp. 18-25, 1993.

[44] A. Furnham and J. Forey, "The attitudes, behaviors and beliefs of patients of conventional vs. complementary (alternative) medicine," Journal of Clinical Psychology, vol. 50, no. 3, pp. 458469, 1994.

[45] A. C. Iversen and P. Kraft, "Does socio-economic status and health consciousness influence how women respond to health related messages in media?" Health Education Research, vol. 21, no. 5, pp. 601-610, 2006.

[46] S. J. Gould, "Consumer attitudes toward health and health care: a differential perspective," Journal of Consumer Affairs, vol. 22, no. 1, pp. 96-118, 1988.

[47] S. J. Gould, "Health consciousness and health behavior: the application of a new health consciousness scale," American Journal of Preventive Medicine, vol. 6, no. 4, pp. 228-237, 1990.

[48] M. Masiero, S. Riva, C. Fioretti, and G. Pravettoni, "Pediatric blood cancer survivors and tobacco use across adolescence and emerging adulthood: a narrative review," Frontiers in Psychology, vol. 7, article 392, 2016.

[49] B. D. Wallston and K. A. Wallston, "Locus of control and health: a review of the literature," Health Education Monographs, vol. 6, no. 2, pp. 107-117, 1978.

[50] B. S. Wallston, K. A. Wallston, G. D. Kaplan, and S. A. Maides, "Development and validation of the health locus of control (HLC) scale," Journal of Consulting and Clinical Psychology, vol. 44, no. 4, pp. 580-585, 1976.

[51] A. Gorini and G. Pravettoni, "An overview on cognitive aspects implicated in medical decisions," European Journal of Internal Medicine, vol. 22, no. 6, pp. 547-553, 2011.

[52] S. Riva, M. Monti, P. Iannello, and A. Antonietti, “The representation of risk in routine medical experience: what actions for contemporary health policy?" PLoS ONE, vol. 7, no. 11, Article ID e48297, 2012.

[53] P. J. Schulz, U. Hartung, and S. Riva, "Causes, coping, and culture: a comparative survey study on representation of back pain in three Swiss language regions," PLoS ONE, vol. 8, no. 11, Article ID e78029, 2013. 

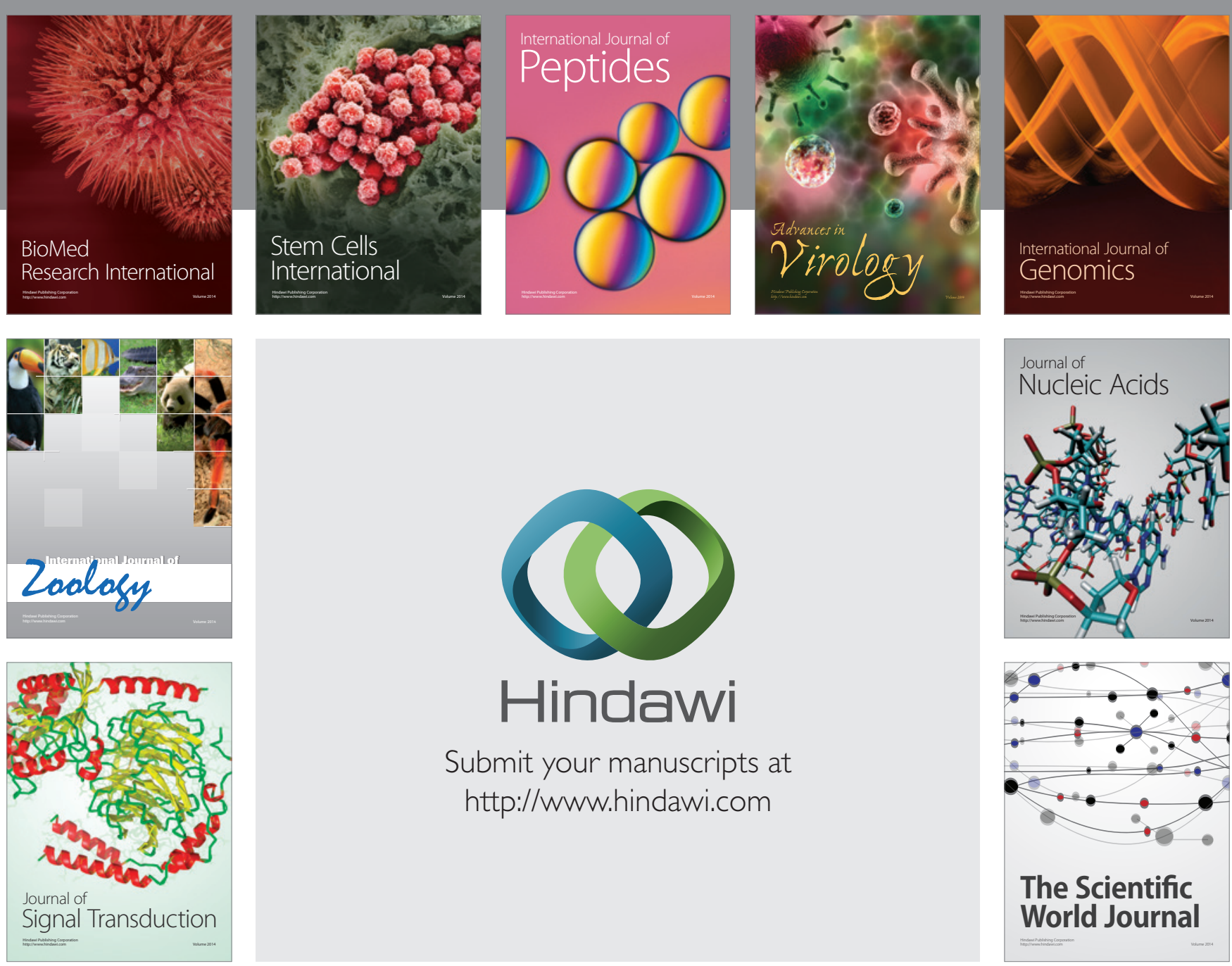

Submit your manuscripts at

http://www.hindawi.com
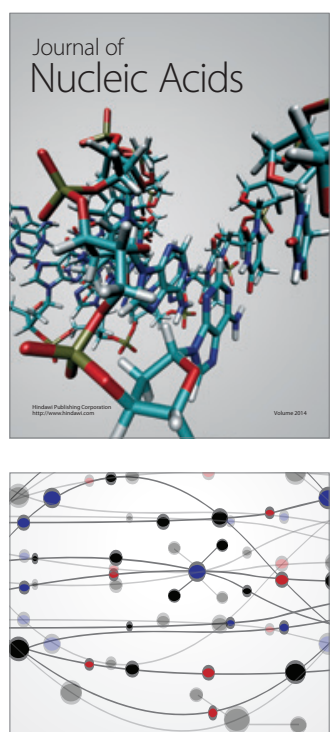

The Scientific World Journal
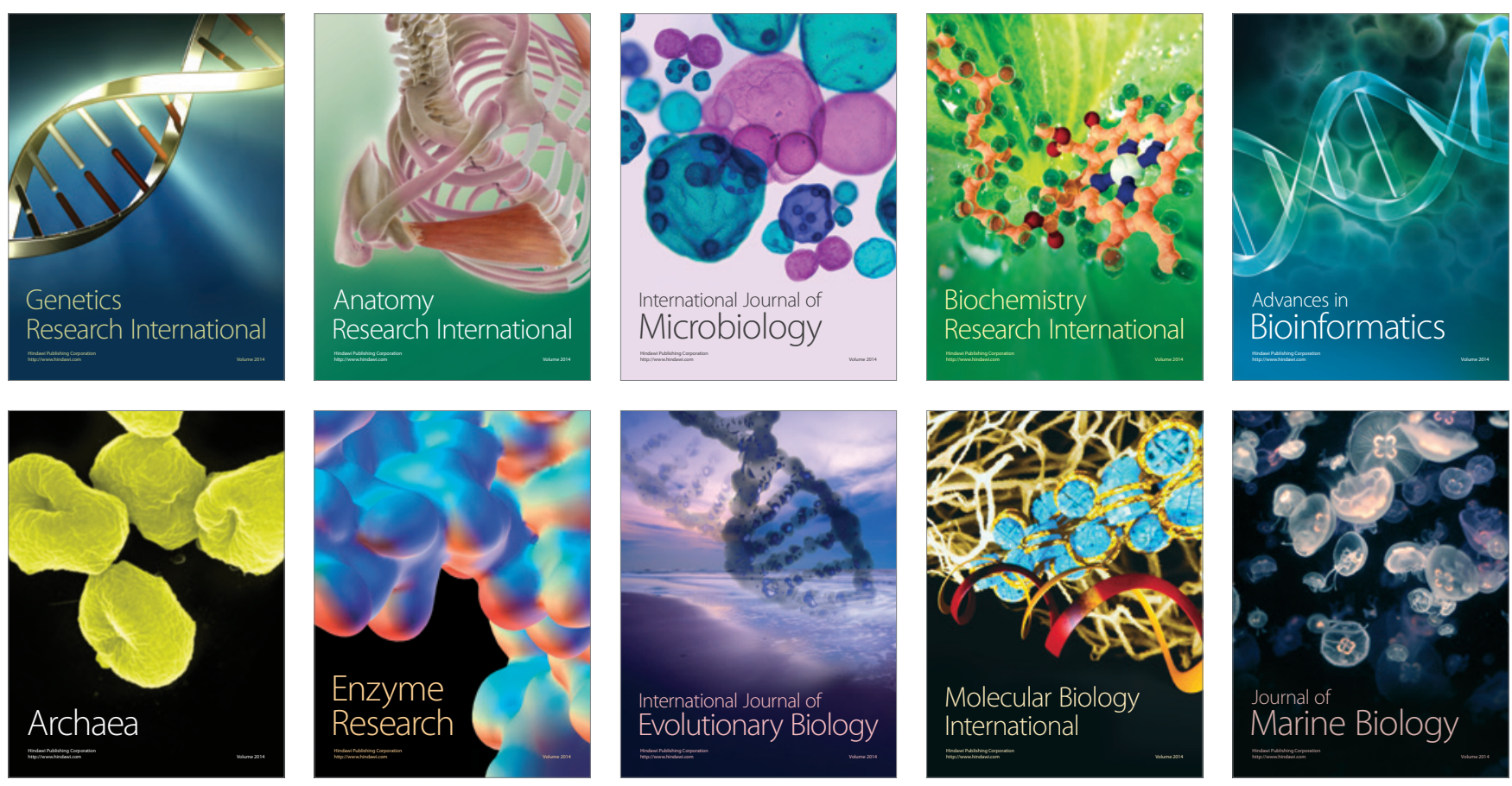